\title{
Preliminary Design of BML: A Behavioral Interface Specification Language for Java Bytecode $^{\star}$
}

\author{
Lilian Burdy, Marieke Huisman ${ }^{1}$, and Mariela Pavlova ${ }^{2, \star \star}$ \\ ${ }^{1}$ INRIA Sophia Antipolis, France \\ ${ }^{2}$ Ludwig-Maximilians-Universität München, German
}

\begin{abstract}
We present the Bytecode Modeling Language (BML), the Java bytecode cousin of JML. BML allows the application developer to specify the behaviour of an application in the form of annotations, directly at the level of the bytecode. An extension of the class file format is defined to store the specification directly with the bytecode. This is a first step towards the development of a platform for Proof Carrying Code, where applications come together with their specification and a proof of correctness. BML is designed to be closely related with JML. In particular, JML specifications can be compiled into BML specifications. We briefly discuss the tools that are currently being developed for BML, and that will result in a tool set where an application can be validated throughout its development, both at source code and at bytecode level.
\end{abstract}

\section{Introduction}

The use of formal methods to show conformance of an implementation w.r.t. a specification has become an accepted technique for the development of securitycritical applications. Various tools exist that allow to specify and validate complex functional or security properties, using different techniques such as runtime assertion checking, testing and verification condition generation. However, often these techniques are restricted to source code level programs, while for many applications, and in particular for mobile code, one needs to be able to also specify and verify the executable (or interpreted) code.

Different possible reasons for this exist: the executable code may not be accompanied by its (specified) source, or one simply does not trust the compiler. And in an attempt to avoid all possible security threats, sometimes securitycritical applications are directly developed at the executable level. Thus, it is essential to have the means to specify and to verify an application directly at this level, without the use of a compiler, and both specification and verification techniques should be tailored directly to the particularities of executable code.

\footnotetext{
* This work is partially funded by the IST programme of the European Commission, under the IST-2003-507894 Inspired project and the IST-2005-015905 MOBIUS project.

** Research done while at INRIA Sophia Antipolis.
} 
Moreover, in order to capture all relevant security requirements, the specification language used should be expressive enough for this.

Proof Carrying Code (PCC) is a typical example where the need to specify and verify executable code directly is imperative, in particular when one wishes to capture complex security policies that cannot be checked with a type checker. PCC is a possible solution to support the secure downloading of applications on a mobile device. The executable code of an application comes together with a specification, and the necessary evidence from which the code client can easily establish that the application respects its specification. In such a scenario, the code producer, who has to produce a correctness proof, will often prefer to do the verification at source code level, and then compile the specification and the proof into the level of executable code. Realising a platform to support this scenario is one of the goals of the MOBIUS project (see http://mobius.inria.fr).

This paper describes the low-level specification language that we propose to specify the security requirements for mobile device applications. Since the most common execution framework for mobile devices is the J2ME platform, our language is tailored to Java bytecode, and thus to the verification of unstructured code. To be able to translate source code level specifications into bytecode level specifications, our specification language is also designed to be closely related to the Java Modeling Language (JML) (see http://www.jmlspecs.org).

Over the last few years, JML has become the de facto specification language for Java source code programs. Different tools exist that allow to validate, verify or generate JML specifications (see [9] for an overview). Several case studies have demonstrated that JML can be used to specify and improve realistic industrial examples (see e.g. [8]). One of the reasons for its success is that JML uses a Java-like syntax. Specifications are written using preconditions, postcondition, class invariants and other annotations, where the different predicates are sideeffect-free Java expressions, extended with specification-specific keywords (e.g. logical quantifiers and a keyword to refer to the return value of a method). Other important factors for the success of JML are its expressiveness and flexibility, and its ability to characterise typical security requirements.

Therefore, we define a variation of JML especially tailored to bytecode, called BML, short for Bytecode Modeling Language. BML supports the most important features of JML. Thus, we can express functional properties of Java bytecode programs in the form of e.g. pre- and postconditions, class and object invariants, and assertions for particular program points like loop invariants. Because of the close connection with JML, JML source code level specifications can be compiled into BML bytecode level specifications without too much difficulty, basically by compiling the source code predicates into bytecode predicates. This allows to do development and verification at source code level, while still being able to ship bytecode level proofs. To the best of our knowledge, no other specification language with similar design goals exists for Java bytecode. Notice that, even though the design of BML was motivated by the need to specify security requirements for mobile device applications, just as JML, BML is a general specification language that can be used for different kinds of applications and analyses. 
Section 2 quickly summarises the relevant features of JML. Section 3 gives a detailed account of BML, describing its syntax and semantics, while Section 4 proposes a format to store BML specifications in a class file. Section 5 discusses the compilation from JML to BML, while Section 6 wraps up and discusses tool support and related and future work.

\section{A Short Overview of JML}

This section gives a short introduction to JML, by means of an example. Throughout the rest of this paper, we assume that the reader is familiar with JML, its syntax and its semantics. For a detailed overview of JML we refer to its reference manual [15. Where necessary, we refer to the appropriate sections of this manual. A detailed overview of the tools which support JML can be found in [9].

To illustrate the different features of JML, Figure 1] shows an example class specification, defining the class Bill. It contains an abstract method round_cost, that computes the cost of a particular round. The method produce_bill is supposed to sum up the costs of the different rounds.

In order not to interfere with the standard Java compiler, JML specifications are written as special comments (tagged with @). Method specifications contain

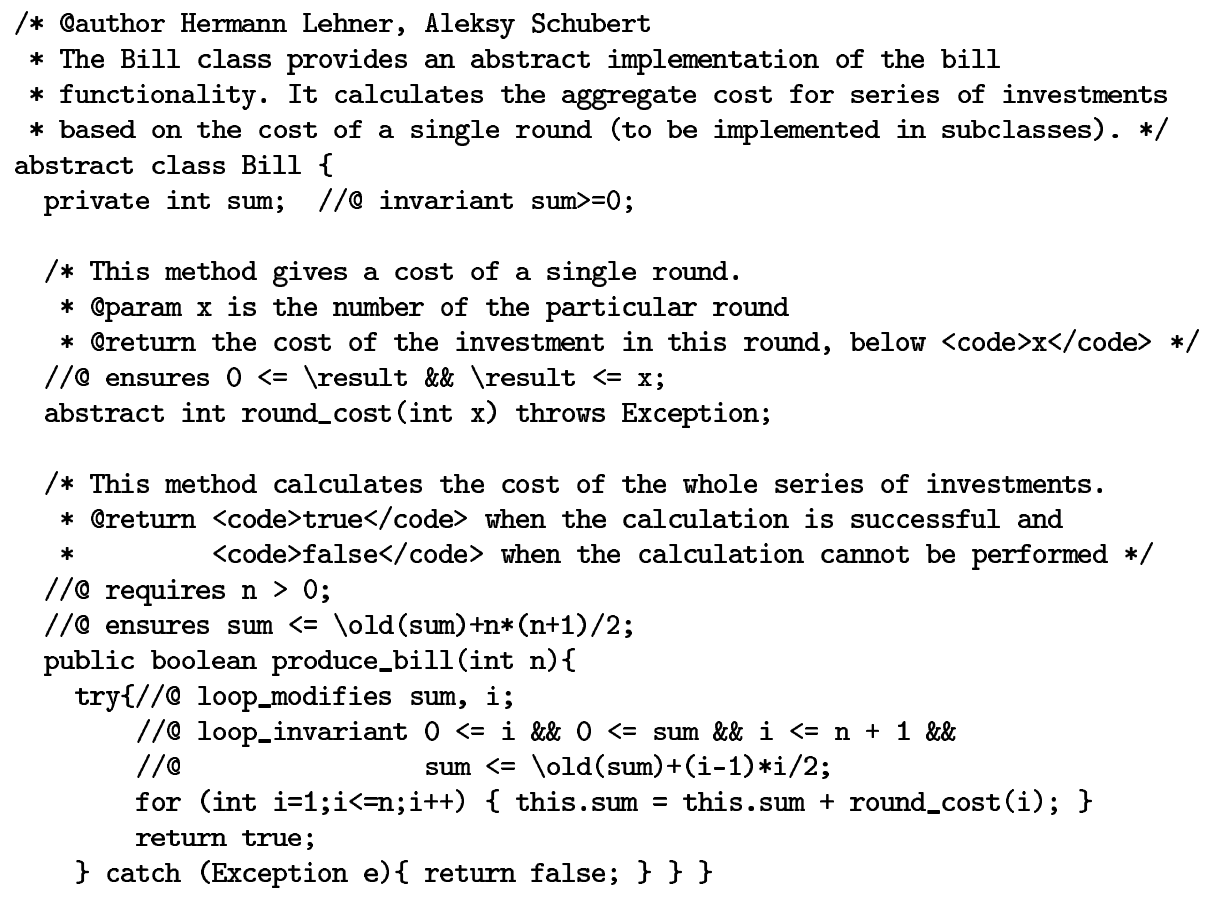

Fig. 1. Class Bill with JML annotations 
preconditions (keyword requires), postconditions (ensures) and frame conditions (assignable). The latter specify which variables may be modified by a method. In a method body, one can annotate all statements with an assert predicate and loops also with invariants (loop_invariant), variants (decreases) and loop frame conditions (loop_modifies). The latter is a non-standard extension of JML, introduced in [11, which we found useful to make program verification more practical. One can also specify class invariants, i.e. properties that should hold in all visible states of the execution, and constraints, describing a relation that holds between any two pairs of consecutive visible states (where visible states are the states in which a method is called or returned from).

The predicates in the different conditions are side-effect free Java boolean expressions, extended with specification-specific keywords, such as \result, denoting the return value of a non-void method, and \old, indicating that an expression should be evaluated in the pre-state of the method.

JML allows to declare special specification-only variables: logical variables (with keyword model) and so-called ghost variables, that can be assigned to in special set annotations.

In Figure 11, the specification for round_cost states that the result of the method should be positive, but less than the number of the round. The specification for produce_bill requires that we compute at least one round, and then ensures an upper-bound on the outcome of the method. We use a loop invariant and loop frame condition to prove the method body correct. Finally, the class invariant specifies that the sum field is always positive.

\section{The Bytecode Modeling Language}

Basically, BML has the same syntax as JML with two exceptions:

1. specifications are not written directly in the program code, they are added as special attributes to the bytecode; and

2. the grammar for expressions only allows bytecode expressions.

Syntax for BML predicates. Figure 2 displays the most interesting part of the grammar for BML predicates, defining the syntax for primary expressions and primary suffixes 1 . Primary expressions, followed by zero or more primary suffixes, are the most basic form of expressions, formed by identifiers, bracketed expressions etc.

Since only bytecode expressions can be used, all field names, class names etc. are replaced by references to the constant pool (a number, preceded by the symbol \#), while registers are used to refer to local variables and parameters. The register $1 \mathrm{v}[0]$ of a non-static method always contains the implicit argument this, the other registers contain the parameters and the local variables declared inside a method body. Compilers often reuse local variable registers throughout the execution of a single method. Thus, when e.g. type checking an annotation

\footnotetext{
${ }^{1}$ See http://www-sop.inria.fr/everest/BML for the full grammar of BML.
} 


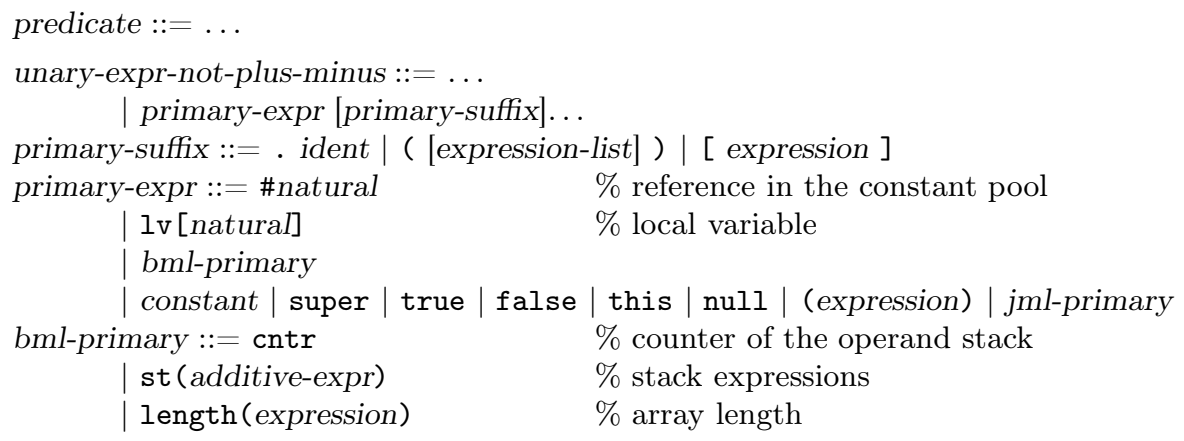

Fig. 2. Fragment of grammar for BML predicates and specification expressions

containing a local variable, it has to be taken into account at which point in the code the annotation is evaluated (but notice that this is not more complicated than reusing the same local variable name in different block statements).

We can use the stack counter (cntr) and stack expressions (st (e), where $e$ is some arithmetic expression) to describe intermediate states of a computation. These are not used in method specifications. We also add a special expression length $(a)$, denoting the length of array $a$. Since the source code expression $a$.length is compiled into a special bytecode instruction arraylength, we also need a special specification construct for this at bytecode level.

In Java source code, one can usually leave the receiver object this implicit. But compilation into bytecode makes this object explicit, i.e. instructions such as putfield always require that the receiver object is loaded on the operand stack. In analogy with this, BML specifications require that the receiver object is written explicitly in expressions (see Figure 3 below).

In JML, many special keywords are preceded by the symbol $\backslash$, to ensure that they will not clash with variable names. For BML, we do not have to worry about this: all variable names are replaced by references to the constant pool or local variable registers. Therefore, the new keywords are written without a special preceding symbol. However, for convenience, we keep the symbol for keywords that are also JML keywords.

At the moment, the use of pure methods is not part of the BML grammar, as there is still ongoing research on the exact semantics of method calls used in specifications. However, we believe that if the theoretical issues have been settled, eventually any tool supporting BML should also support thi:2.

Class and method specifications. BML contains equivalent constructs for all specification constructs of JML Level 0 (see [15, §2.9]), which defines the features that should be understood and checked by all JML tools. It also contains several constructs from JML level 1, that we find important to be able to write meaningful

${ }^{2}$ In fact, we think that both at source code and at bytecode level, specifications will benefit significantly from being allowed to use method calls in them. 
specifications for the example applications studied in the MOBIUS project, namely static invariants; object and static constraints; and loop variants.

We choose to keep the notion of loop specification in BML, even though there is no high level loop construct in bytecode. But to be able to prove termination, one needs to prove decrease of a loop variant, which makes the treatment of loops different from the treatment of other statements. Also, experiences with verification of realistic case studies have shown that it is beneficial to know which variables may be modified by the code block that corresponds to the loop. For this, we use the special clause loop_modifies. This allows to write concise specifications, and to efficiently generate proof obligations using a weakest precondition calculus. Moreover by keeping the notion of loop specification explicit in BML, we keep the correspondence with JML specifications more direct.

As mentioned above, specifications are stored as special attributes in the class file. This means that every class contains a table with invariant and constraint annotations, while each method has extra attributes containing its specifications. Finally, the code for the method body is annotated with local annotation tables for the assert annotations and the loop specifications. Section 4 defines the precise format of these attributes.

Since the bytecode and BML specifications are two separate entities, they should be parsed independently. Concretely this means that the grammar of BML is similar to the grammar of type specifications, method specifications and data groups of JML [15, §A.5, A.6, A.7], restricted to the constructs in JML level 0, plus the constructs of JML level 1 mentioned, but with the changes to the grammar for predicates and specification expressions, as mentioned above.

An example BML specification. To show a typical BML specification, Figure 3 presents the BML version of the JML specification of method produce_bill in Figure 1. Notice that the field sum has been assigned the number 24 in the constant pool, and that it is always explicitly qualified with $1 \mathrm{v}$ [0] (denoting this). Further, lv[1] denotes the parameter n, while lv[2] denotes the local variable $i$.

The class invariant gives rise to the following BML specification (stored in the class file as a special user-specific attribute, as explained below):

invariant: $\# 24>=0$

This expression is not qualified with $1 \mathrm{v}[0]$, as it is implicitly quantified over all objects that are an instance of a subclass of class Bill ( $c f$. the JML semantics [15, $\S 8.2])$.

Structural and typing constraints for BML specifications. BML specifications have to respect several structural and typing constraints, similar to the structural and typing constraints that the bytecode verifier imposes over the class file format. Examples of typing constraints that a BML specification must respect are the following: 


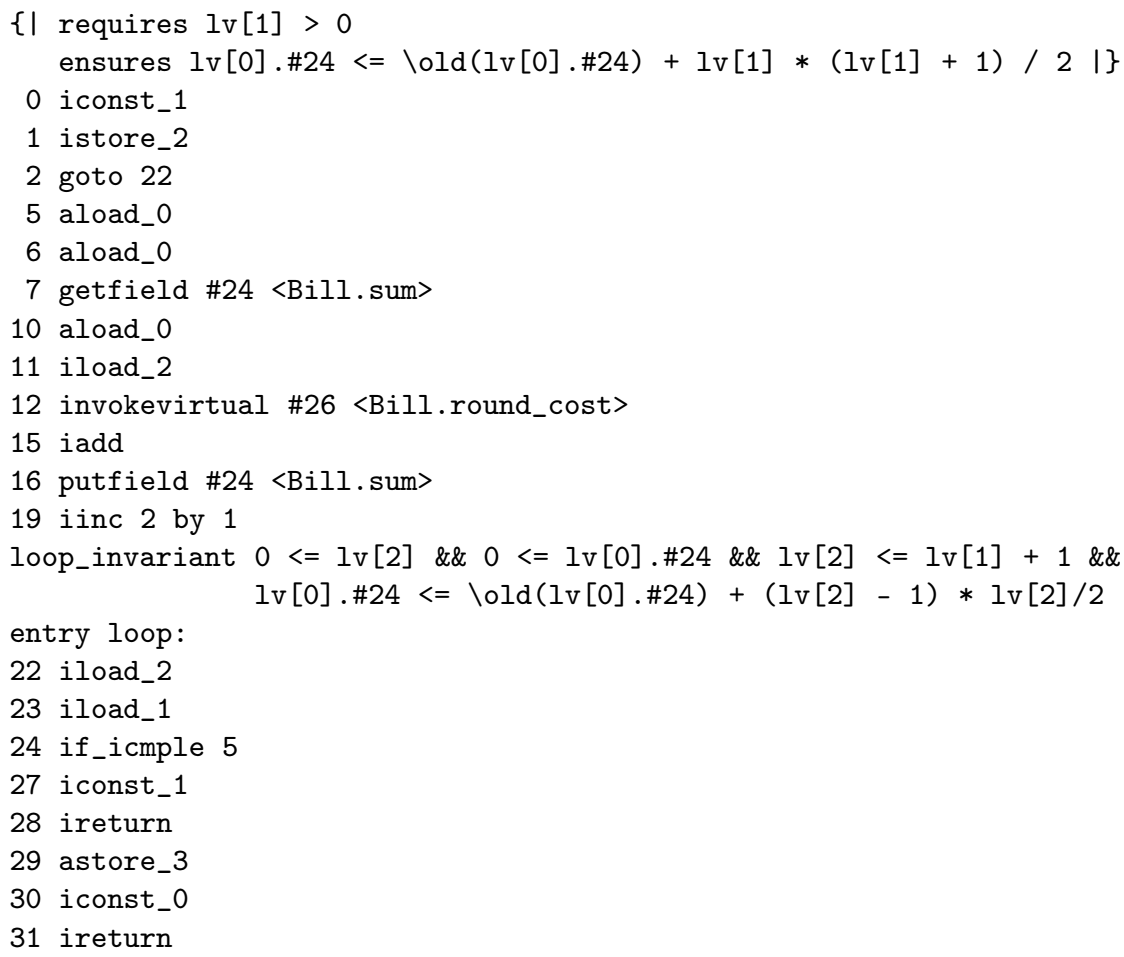

Fig. 3. Bytecode + BML specification for method produce_bill in class Bill

- field access expression $e$.ident is well-typed only if $e$ is of a subtype of the class where the field described by the constant pool element at index ident is declared;

- array access expression $e_{1}\left[e_{2}\right]$ is well-typed only if $e_{1}$ is of array type and $e_{2}$ is of integer type; and

- predicate $e_{1}<: e_{2}$ is well-typed only if the expressions $e_{1}$ and $e_{2}$ are of type java.lang. Class (which is the same as the JML type \TYPE ).

Examples of structural constraints that a BML specification must respect are the following:

- all references to the constant pool must be to an entry of the appropriate type; for example, for field access expression $e$. ident, ident must reference a field in the constant pool; while for expression \type (ident), ident must be a reference to a constant class in the constant pool;

- every ident in a BML specification must be a correct index in the constant pool; and

- if the expression $1 \mathrm{v}[i]$ appears in a BML method specification, $i$ must be a valid index in the method's local variables table.

These checks are best implemented as an extension of the bytecode verifier. 
Semantics of BML expressions. The semantics of BML specifications follows the semantics of JML specifications [15]. But, just as a JML specification can be mapped into a more fundamental Hoare triple specification, we can also define a semantics for BML in terms of a basic logic for Java bytecode, namely the so-called MOBIUS base logic. This logic will be the core of the PCC platform developed within the project. This logic (see [7] for an earlier version, without exceptions) has been proven sound in Coq w.r.t. a formalisation of the virtual machine. On top of this, a direct verification condition generator has been proven sound, also in Coq. And, as a first step towards efficient tool development, a translation of bytecode into guarded commands has been defined and proven correct, w.r.t. verification condition generation [16].

Defining the mapping of BML specifications into this MOBIUS base logic is defined in two steps. First the evaluation of predicates is defined over the program state (i.e. over the heap, store and operand stack), and second the complete BML specifications are translated into judgements of the MOBIUS base logic. Notice that this embedding allows to use the verification condition generator for the MOBIUS base logic also for BML specifications.

Judgements in the MOBIUS base logic are of the form $\mathrm{G}, \mathrm{Q} \vdash\{A\} p c\{B\}(I)$, where $\mathrm{G}$ is a proof context, and $\mathrm{Q}$ the local annotation table, i.e. the table that associates assert annotations with particular instructions. Further, $A$ is a (local) precondition, relating the state at label $p c$ with the initial state, while $B$ is a (local) postcondition, relating the initial, current and final state, and $I$ is a (local) invariant, i.e a predicate that is supposed to hold throughout execution of the current method.

Mapping class specifications (invariants and constraints) and method specifications into the MOBIUS base logic is straightforward. Since the MOBIUS base logic only has one postcondition, the normal and exceptional postconditions are combined into a single postcondition, specifying with a case distinction which conditions should hold if the state is normal or exceptional, respectively. Frame conditions are also added to the postconditions, specifying explicitly which variables are allowed to be changed. Since predicates in the MOBIUS base logic specify properties over the whole heap, this can be expressed directly: all locations that are not mentioned in the frame condition of the method (evaluated in the pre-state of the method) should be unchanged. Methods with multiple specifications are translated only after desugaring them into a single method specification $c f$. [20].

Assert and set statements are inserted directly in the local annotation table 3 . However, for loop specifications some manipulations are necessary to produce the appropriate assert annotations, due to the unstructured nature of bytecode. The loop invariants can be added directly to the local annotation table, but loop variants and loop frame conditions first are transformed into a sequence of assert and set annotations (after introducing appropriate ghost variables).

${ }^{3}$ In fact, at the moment, the MOBIUS base logic does not support ghost variables; but these will be added in the near future. 
This transformation is done at the level of BML, after which we can add the annotations to the local annotation table.

The transformation of the loop variant basically proceeds as follows. Let variant be the expression declared in the decreases clause. We declare ghost variables loop_init (initially set to true) and loop_variant (whose initialisation is not essential). If $l$ is the program point where we enter the loop, then at that point we add an assertion

\section{//@ assert !loop_init ==> $(0<=$ variant \&\& variant < loop_variant $)$;}

followed by:

//@ set loop_init $=$ false; set loop_variant $=$ variant;

This ensures that every time the loop entry point $l$ is reached again, the decrease of the loop variant is checked. Only a path that goes through the loop can set loop_init to false.

For transforming loop frame conditions, we use again that in the MOBIUS base logic we can express properties of the heap. We make a transformation into a sequence of assert and set statements, declaring ghost variables to remember the old heap and all locations mentioned in the loop frame condition, and a ghost variable loop_init as above. Then we assert at the entry point of the heap that if loop_init does not hold, any location that is not mentioned in the loop frame condition should remain unchanged. Notice that this assertion cannot be directly expressed in BML, but it can be expressed in the MOBIUS base logic. Finally, in the MOBIUS base logic we add appropriate ghost variable updates to remember the old heap and the locations of the loop frame condition when the loop was first entered.

\section{Encoding BML Specifications in the Class File Format}

To store BML specifications together with the bytecode it specifies, we encode them in the class file format. Recall that a class file contains all the information related to a single class or interface, e.g. its name, which interfaces it implements, its super class and the methods and fields it declares. The Java Virtual Machine Specification [17] prescribes the mandatory elements of the class file: the constant pool, the field information and the method information. The constant pool is used to construct the runtime constant pool upon class or interface creation. This will serve for loading, linking and resolution of references used in the class. The JVM specification allows to add user-specific information to the class file ([17, §4.7.1]) as special user-specific attributes. We store BML specifications in such user-specific attributes, in a compiler-independent format 4 . To ensure that the augmented class files are executable by any implementation of the JVM, the user-specific attributes cannot be inserted in the list with bytecode

${ }^{4}$ Another possibility would be to use metadata to encode the specifications, but this is only supported in Java 1.5, and it is (currently) not directly compatible with JML. 

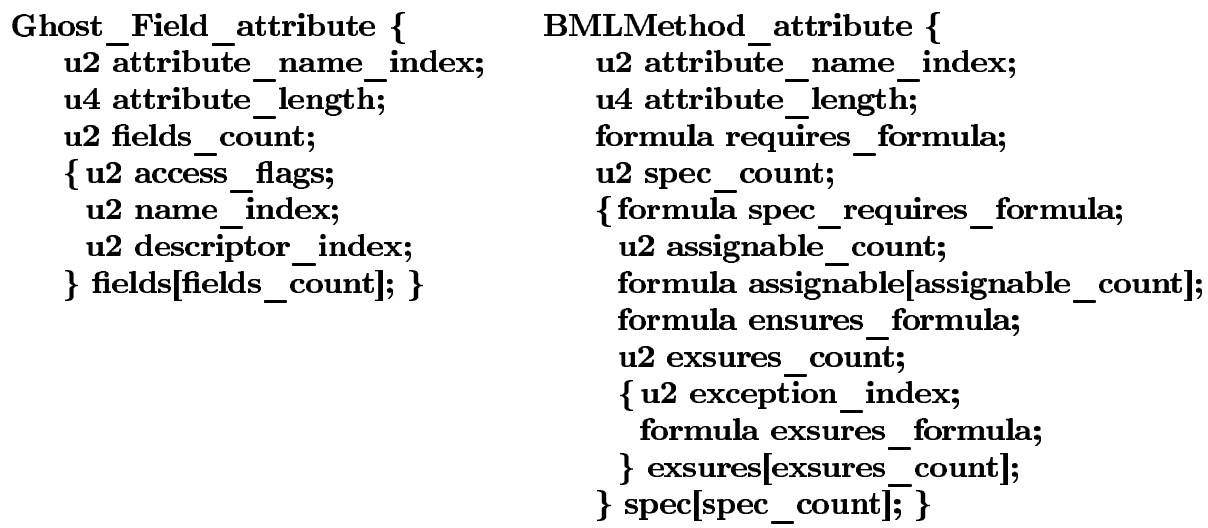

Fig. 4. Attributes for ghost field declarations and method specifications

instructions. Instead BML annotations are stored separately from the method body, and where necessary the annotations contain the index of the instruction that they specify. The use of special attributes ensures that the presence of BML annotations does not have any impact on the application's performance, i.e., the augmented class file should not slow down loading or normal execution of the application. requirement is important for mobile.

For each class, we add the following information to the class file:

- a second constant pool which contains constant references for the BML specification expressions;

- an attribute with the ghost fields used in the specification;

- an attribute with the model fields used in the specification;

- an attribute with the class invariants (both static and object); and

- an attribute with the constraints (both static and object).

Apart from the second constant pool, all extra class attributes basically contain the name of the attribute, the number of elements it contains, and a list with the actual elements.

If a model or a ghost field is dereferenced in the specification, then a constantFieldRef is added to the second constant pool as the Java compiler does for any dereferenced Java field in the original constant pool of the class. Note that in this way, the BML encoding will not affect the JVM performance. In particular, if we would use the original constant pool for storing constants originating from specifications, the search time in the original constant pool might degrade significantly (especially for a large specification).

The left-hand column of Figure 4 shows the format of the ghost fields attribute. This should be understood as follows: the name of the attribute is given as an index into the constant pool. This constant pool entry will be representing a string "Ghost_Field". Next we have the length of the attribute, which should be $2+6 *$ fields_count (the number of fields stored in the list). The fields 
table stores all ghost fields. For each field we store its access flags (e.g. public or private), and the name index and descriptor index, both referring to the constant pool. The first must be a string, representing the (unqualified) name of the variable, the latter is a field descriptor, containing e.g. type information. The tags $\mathbf{u} 2$ and $\mathbf{u} 4$ specifies the size of the attribute, 2 and 4 bytes, respectively. The model field attribute is similar.

In a similar way, we define attributes for class invariants and constraints, containing a list of invariants and constraints, respectively. These contain the predicate, a tag whether the invariant (constraint) is defined over instances or static, and appropriate visibility modifiers.

The JVM specification prescribes that the attribute with method information at least contains the code of each method. We add attributes for the method specification, set statements, assert statements, and loop specifications.

The attribute for lightweight behaviour specifications is shown in the righthand column of Figure 4 (heavyweight behaviour specifications are handled similarly). The global requires formula is the disjunction of all preconditions in the different specification cases of the method. For each specification case, we then have a precondition (spec_requires_formula), a list of assignable expressions, a postcondition (ensures formula) and a list of exceptional postconditions (stored in the exsures attribute). If a clause is not explicitly specified, its default value will be stored here.

The attributes for set and assert statements and loop specifications have the same format as e.g. the ghost variable attribute: a length entry and a list of elements. The elements storing set and assert statements do not only keep the appropriate predicate or expression, but also an index to the appropriate point in the bytecode. Similarly, the elements for loop specifications contain the loop specification (invariant, variant and frame conditions), plus an index to the bytecode instruction that corresponds to the entry of the loop. If the specification does not contain a loop variant, we indicate this, using a special tag for the decreases clause.

\section{Compiling JML Specifications into BML Specifications}

Since it is often easier and more intuitive to specify and verify at source code level, we have defined a compiler from JML to BML: JML2BML. BML is designed to be very close to JML, so the correspondence between the original and the compiled specification is straightforward. Notice that in principle, the same can be done for the proofs, i.e. a source code level proof can be compiled into a bytecode level proof. It is future work to define this in full detail, but some work in this direction has already been done [6].

The JML specification is compiled separately from the Java source code. In fact, JML2BML takes as input an annotated Java source file and the Java class file produced by a non-optimising compiler with the debug flag set.

From the debug information, we use in particular the Line_Number_Table and the Local_Variable_Table attributes. The presence of these attributes is optional [17], but almost all standard non-optimising compilers can generate 
them. The Line_Number_Table links line numbers in the Java source code with the Java bytecode instructions. The Local_Variable_Table describes the local variables that appear in a method.

To compile loop invariants appropriately, the control flow graph corresponding to the list of bytecode instructions resulting from the compilation of a method body must be a reducible control flow graph, i.e. every cycle in the graph must have exactly one entry point (see [1]). Note that this is not a serious restriction; all non-optimising Java compilers produce reducible control flow graphs and in practice even most hand-written bytecode is reducible.

The compilation from JML specifications into BML is defined in several steps. As mentioned above, we assume that the Java source code has been compiled with the debug flag set, and that we have access to the generated class file.

Compilation of ghost and model field declarations. Ghost and model variables declared in the specification are compiled into the special class attributes that contain all ghost variable and model variable declarations.

Linking and resolving of source data structures. The JML specification is transformed into an intermediate format, where the identifiers are resolved to their corresponding data structures in the class file. The Java and JML source identifiers are linked to their identifiers on bytecode level, i.e. the corresponding indexes either from the second constant pool or from the Local_Variable_Table attribute. This is similar to the linking and resolving stage of the Java source code compiler.

Locating instructions for annotation statements. Annotation statements, like loop specifications and asserts are associated with the appropriate point in the bytecode program, using the Line_Number_Table attribute.

A problem is that a source line may correspond to more than one instruction in the Line Number Table. This makes it complicated to identify the exact loop entry instruction in the bytecode, and thus to know to which instruction the compiled loop specification should be associated. To solve this, we use the following heuristics: if the control flow graph of the bytecode is reducible and we search from an index in the Line_Number Table that corresponds to the first line of a source loop, then the first loop entry instruction found will be the loop entry corresponding to this source loop. We do not have a formal correctness proof for this algorithm, because it depends on the particular implementation of the compiler, but experiments show that the heuristic works successfully for Sun's non-optimising Java compiler.

Compilation of JML predicates. JML predicates are Java boolean expressions. However, the JVM does not provide direct support for several integral types, such as byte, short, char, or for booleans. Instead, they are encoded as integers. Therefore, the compiler wraps up the boolean expressions in the JML specification by a conditional function, returning 1 if the predicate is true, 0 otherwise.

Generation of user-specific class attributes. Finally, the complete specification is compiled into appropriate user-specific attributes, using the format defined in the previous section. 


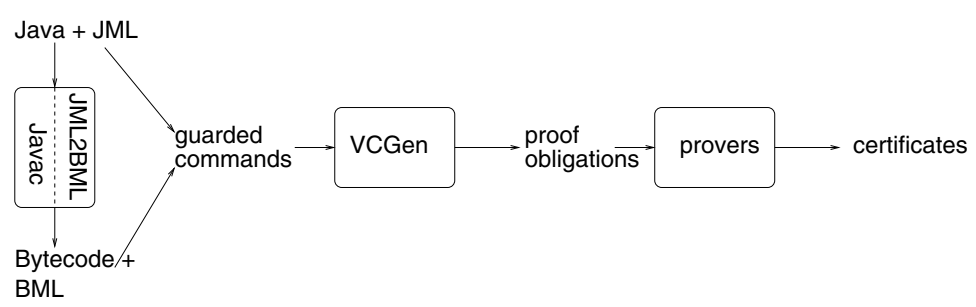

Fig. 5. Overview of MOBIUS tool set

\section{Conclusions and Related Work}

This paper presents the Bytecode Modeling Language (BML). BML allows one to specify and verify an application directly at the level of bytecode. Its syntax and semantics are directly inspired by the source code level specification language JML. The possibility to reason direct at the level of bytecode, without relying on a compiler, is of major importance for guaranteeing the security of applications (for example in a context of mobile code, where some applications are written in bytecode directly, to avoid security problems related with compilation). However, to make such verifications tractable, it is important that the specification language is intuitive and provides a sufficient degree of abstraction, without the need to talk too much about the internal structure of the state (heap, store etc.). BML does exactly this: it is designed to be close to the source code level specification language JML and provides a high level of abstraction. It is designed for program verification, and its semantics supports the development of a verification condition generator for unstructured code. Moreover, because of its close connection with JML, it is not too complicated to compile source code level specification into bytecode level specifications. The BML language as we have defined it now, corresponds roughly to JML level 0, i.e. that part of JML whose semantics is relatively well understood. However, more advanced constructs of JML can be easily added to BML, if required.

Tool support. As part of the MOBIUS project, we plan to develop a program verification tool set that supports both JML and BML. Figure 5 outlines the general architecture of this tool set. Thus, both Java/JML and bytecode/BML can be used as input application. Annotated programs are translated into a guarded command format, for which an appropriate verification condition generator is used to generate proof obligations that can be discharged with a theorem prover (either automatic or interactive). To support the PCC platform, the provers will be instrumented to produce certificates. In addition, source code applications annotated with JML can be compiled into bytecode annotated with BML.

The development of the JML subcomponent of the tool set will be based on experiences with ESC/Java [13] and JACK [11]. Several tools and algorithms 
(notably the compiler and the verification condition generator) for BML have already been implemented, see [1019], but more work is needed to cover the whole language. Moreover, to make the tool set usable in practice, we will also need a tool to inspect and write BML specifications directly, and a run-time checker for BML specifications. The latter can be implemented by a code transformation, inserting explicit run-time checks in the bytecode, or by extending the virtual machine to take the user-specific attributes with specifications into account. It is also important to have tool support for checking the structural and typing constraints for BML specifications. Such a tool can be built as an extension the Java bytecode verifier.

Our initial experiments with compilation of specifications has shown that there exists indeed a correspondence between the proof obligations generated at source and at bytecode level, modulo differences in elimination of trivial goals, handling of boolean expressions, and the naming convention of generated variables [19]. Moreover, when the proofs are done with the Coq prover, different names are generated for hypotheses at source code and bytecode level. It is future work to clean up the compilation, so there is a one-to-one correspondence.

Related work. The interest in specification and verification of bytecode applications is quite recent, and not too much work has been done in that direction. Several logics have been developed to reason about bytecode, e.g. by Bannwart \& Müller 4 and within the MRG project 3. However, in this work the main focus was the development of a sound proof system, while the focus of BML is to write understandable specifications for bytecode. JVer is a tool to verify annotated bytecode [12. However, as specification language they use a subset of JML, i.e. a source code level specification language.

The development of BML is clearly inspired by the development of the JML specification language [15]. Both JML and BML follow the Design by Contract principle introduced first in Eiffel [18]. The Boogie project [5] introduces in similarly the Design by Contract principles into the $\mathrm{C} \#$ programming language, both at source code level and for CIL, the .NET intermediate language. The possibility to check a property at run-time, using the assert construct, has been long adopted in the $\mathrm{C}$ programming language and recently also in Java (Java 1.5, see [14, §14.10]).

Finally, we should mention the Extended Virtual Platform project5. This project aims at developing a framework that allows to compile JML annotations, to allow run-time checking 2]. However, in contrast to our work, they do not intend to do static verification of bytecode programs. Moreover, their platform takes JML-annotated source code files as starting point, so it is not possible to annotate bytecode applications directly.

Acknowledgements. We thank Lennart Beringer and Olha Shkaravska for discussions about the semantics of BML.

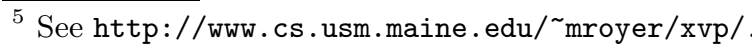




\section{References}

1. A.V. Aho, R. Sethi, and J.D. Ullman. Compilers: Principles, Techniques, and Tools. Addison-Wesley, 1986.

2. S. Alagić and M. Royer. Next generation of virtual platforms. Article in odbms .org, 2005. Available from http://odbms.org/about_contributors_alagic.html.

3. D. Aspinall, L. Beringer, M. Hofmann, H.-W. Loidl, and A. Momigliano. A program logic for resource verification. In K. Slind, A. Bunker, and G. Gopalakrishnan, editors, Theorem Proving in Higher Order Logics (TPHOLs'04), volume 3223 of LNCS, pages 34-49. Springer, 2004.

4. F.Y. Bannwart and P. Müller. A logic for bytecode. In F. Spoto, editor, Bytecode Semantics, Verification, Analysis and Transformation (BYTECODE), volume 141 of ENTCS, pages 255-273. Elsevier, 2005.

5. M. Barnett, B.-Y. E. Chang, R. DeLine, B. Jacobs, and K.R.M. Leino. Boogie: A modular reusable verifier for object-oriented programs. In Formal Methods for Components and Objects (FMCO '05), LNCS. Springer, 2005.

6. G. Barthe, T. Rezk, and A. Saabas. Proof obligations preserving compilation. In R. Gorrieri, F. Martinelli, P. Ryan, and S. Schneider, editors, Proceedings of FAST'05, volume 3866 of $L N C S$, pages 112-126. Springer, 2005.

7. L. Beringer and M. Hofmann. A bytecode logic for JML and types. In ASIAN Symposium on Programming Languages and Systems (APLAS 2006), 2006.

8. C. Breunesse, N. Cataño, M. Huisman, and B. Jacobs. Formal methods for smart cards: an experience report. Science of Computer Programming, 55:53-80, 2005.

9. L. Burdy, Y. Cheon, D. Cok, M. Ernst, J. Kiniry, G.T. Leavens, K.R.M. Leino, and E. Poll. An overview of JML tools and applications. STTT, 7(3), 2005.

10. L. Burdy and M. Pavlova. Java bytecode specification and verification. In L.M. Liebrock, editor, proceedings of $S A C^{\prime} 06$. ACM, 2006.

11. L. Burdy, A. Requet, and J.-L. Lanet. Java Applet Correctness: a DeveloperOriented Approach. In Formal Methods (FME'03), number 2805 in LNCS, pages 422-439. Springer, 2003.

12. A. Chander, D. Espinosa, N. Islam, P. Lee, and G. Necula. JVer: A Java Verifier. In Proceedings of the Conference on Computer Aided Verification (CAV'05), 2005.

13. D. Cok and J.R. Kiniry. ESC/Java2: Uniting ESC/Java and JML. In G. Barthe, L. Burdy, M. Huisman, J.-L. Lanet, and T. Muntean, editors, CASSIS, volume 3362 of LNCS, pages 108-128. Springer, 2004.

14. J. Gosling, B. Joy, G. Steele, and G. Bracha. The Java Language Specification, Third Edition. Sun Microsystems, Inc., 2005.

15. G.T. Leavens, E. Poll, C. Clifton, Y. Cheon, C. Ruby, D. Cok, P. Müller, and J. Kiniry. JML reference manual. http://www.cs.iastate.edu/ leavens/ JML/jmlrefman/jmlrefman_toc.html, 2005.

16. H. Lehner and P. Müller. Formal translation of bytecode into BoogiePL, 2007.

17. T. Lindholm and F. Yellin. The Java ${ }^{\text {TM }}$ Virtual Machine Specification. Second Edition. Sun Microsystems, Inc., 1999.

18. B. Meyer. Object-Oriented Software Construction. Prentice Hall, $2^{\text {nd }}$ rev. edition, 1997.

19. M. Pavlova. Specification and verification of Java bytecode. $\mathrm{PhD}$ thesis, Université de Nice Sophia-Antipolis, 200x.

20. A.D. Raghavan and G.T. Leavens. Desugaring JML method specifications. Technical Report TR \#00-03e, Department of Computer Science, Iowa State University, 2000. Current revision from May 2005. 